\title{
Romosozumab or alendronate for fracture prevention in East Asian patients: a subanalysis of the phase III, randomized ARCH study
}

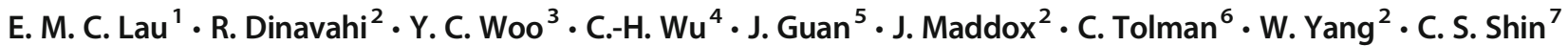

Received: 20 June 2019 / Accepted: 27 January 2020 / Published online: 11 February 2020

(C) The Author(s) 2020

\begin{abstract}
Summary Romosozumab, a sclerostin antibody, exerts dual effect to increase bone formation and decrease bone resorption. Among high-risk postmenopausal East Asian women, romosozumab followed by alendronate was associated with lower incidences of fractures vs alendronate alone. Romosozumab demonstrates potential to address an unmet need in osteoporosis management in Asia.

Introduction Romosozumab, a sclerostin antibody, exerts dual effect to increase bone formation and decrease bone resorption. The global ARCH study demonstrated superiority of romosozumab followed by alendronate in reducing fracture risk in high-risk postmenopausal osteoporotic women vs alendronate alone. We report outcomes among ARCH East Asian patients.

Methods In ARCH, 4093 postmenopausal osteoporotic women with fragility fracture were randomized 1:1 to monthly romosozumab $210 \mathrm{mg}$ or weekly alendronate $70 \mathrm{mg}$ for $12 \mathrm{months}$, both followed by open-label alendronate. Primary endpoints were incidence of new vertebral fracture $(\mathrm{VF})$ at 24 months and clinical fracture at primary analysis (confirmed fractures in $\geq 330$ patients and all patients had opportunity to attend month 24 visit). This post hoc analysis was not powered to detect fracture-rate differences.

Results This analysis included 275 patients from Hong Kong, Korea, and Taiwan. Romosozumab followed by alendronate reduced risk of new VFs at 24 months by $60 \%(P=0.11)$ and clinical fractures at primary analysis by $44 \%(P=0.15)$ vs alendronate alone. Romosozumab followed by alendronate significantly increased mean bone mineral density at 24 months from baseline by a further $9.0 \%, 3.3 \%$, and $3.0 \%$ at the lumbar spine, total hip, and femoral neck vs alendronate alone. Adverse event (AE) rates, including positively adjudicated serious cardiovascular AEs (1.6\% vs $1.4 \%$ at 12 months for romosozumab vs alendronate), were similar across treatment groups.

Conclusions Consistent with the global analysis, romosozumab followed by alendronate was associated with lower incidences of new vertebral, clinical, non-vertebral, and hip fractures vs alendronate alone among East Asian patients.
\end{abstract}

Keywords Asia $\cdot$ Fragility fracture $\cdot$ Imminent risk $\cdot$ Osteoporosis $\cdot$ Romosozumab $\cdot$ Treatment

\section{Introduction}

Osteoporosis and its associated fragility fractures are debilitating conditions that result in significant morbidity and mortality

E. M. C. Lau

edith.lau@hkosc.org

1 Hong Kong Orthopaedic and Osteoporosis Center for Treatment and Research, 6th Floor, Tower 2, New World Tower, 18 Queen's Road Central, Hong Kong, Hong Kong

2 Amgen, Inc., Thousand Oaks, CA, USA

3 Department of Medicine, University of Hong Kong, Queen Mary Hospital, 102 Pok Fu Lam Road, Pok Fu Lam, Hong Kong worldwide [1]. Fragility fractures are associated with decline in physical and cognitive function, high rates of disability, loss of independence [2], and increased mortality — up to one in four patients in Asia who sustain a hip fracture die within a year, and

4 Department of Family Medicine, National Cheng Kung University Hospital, College of Medicine, National Cheng Kung University, Sheng Li Road, Tainan, Taiwan

5 UCB Pharma, Brussels, Belgium

6 Amgen Asia Holding Ltd, One Island East, 18 Westlands Rd, Quarry Bay, Hong Kong

7 Department of Internal Medicine, Seoul National University College of Medicine, Seoul, South Korea 
less than half of those who survive regain their previous level of function [3, 4]. Importantly, patients who sustain a fracture are at imminent risk of a future fracture, defined as risk of near-term fracture within the next 12-24 months [5-7]. In Asia, 6-8\% of hip fracture patients suffer another fracture within 2 years, with second fractures occurring as early as 4 weeks after the first event [8-10].

As a result of the exponentially aging population in Asia, it is projected that by 2050 , more than half of the world's fractures and an estimated 2.6 million hip fractures will occur in the region $[11,12]$. Despite this, osteoporosis remains alarmingly underdiagnosed and undertreated in Asia, even among patients who have already sustained a fragility fracture. In a study of over 1000 postmenopausal women with hip fractures from seven countries in Asia, only one in three underwent bone mineral density (BMD) testing and received osteoporosis medications after discharge [13].

Oral bisphosphonates, particularly alendronate, are the most commonly prescribed anti-osteoporosis medication in the region [14-16]; however, two out of three patients in Asia are non-adherent to oral bisphosphonates within 312 months of prescription [17-19]. As a result of the high burden and proportion of patients with imminent risk of fracture in the region, there is a strong need for more potent medications that can impact outcomes early in the course of the disease [20, 21].

Romosozumab is a bone-forming agent and monoclonal antibody that binds and inhibits sclerostin, with a dual effect of increasing bone formation and decreasing bone resorption $[22,23]$. Its unique dual mechanism of action offers more potent and rapid improvement in bone mass, structure, and strength to reduce the risk of fractures, differentiating it from other anti-osteoporosis medications such as antiresorptives and parathyroid hormone analogues that decrease and increase, respectively, both bone formation and resorption [24]. In the phase III FRActure study in postmenopausal woMen with ostEoporosis (FRAME; NCT01575834), romosozumab was associated with a lower risk of vertebral fracture than placebo at 12 months and, after transition to denosumab, at 24 months [25]. On the basis of this trial, romosozumab was approved in Japan in January 2019 for the treatment of osteoporosis in patients at high risk of fracture [26].

The phase III Active-contRolled fraCture study in postmenopausal women with osteoporosis at High risk (ARCH) was the first registrational head-to-head study to demonstrate superior antifracture efficacy of a novel osteoporosis medication, romosozumab, over alendronate, the current standard of care [27]. Compared with alendronate treatment alone for 24 months, romosozumab treatment in the first year followed by alendronate in the second year significantly reduced the risk of new vertebral, clinical, non-vertebral, and hip fractures in postmenopausal women at high fracture risk [27]. In ARCH, approximately $7 \%$ of global enrollment was from the Asia Pacific region. In this post hoc analysis of the ARCH trial, we evaluated the efficacy and safety of romosozumab followed by alendronate compared with alendronate alone in patients from East Asia.

\section{Methods}

\section{Study design and patients}

$\mathrm{ARCH}$ was a phase III, multicenter, international, randomized, double-blind trial. Full details of the study design and eligibility criteria have been previously reported [27]. Briefly, ambulatory postmenopausal women aged 55-90 years with severe osteoporosis, from 41 countries globally, were randomly assigned (1:1) to monthly subcutaneous romosozumab $210 \mathrm{mg}$ (Amgen, Inc., Thousand Oaks, CA, USA, and UCB Pharma, Brussels, Belgium) or weekly oral alendronate $70 \mathrm{mg}$ (Merck \& Co., Inc., Kenilworth, NJ, USA) for 12 months, followed by open-label alendronate in both groups. Patients also received daily calcium and vitamin D supplements. Patients had a BMD T-score of -2.5 or lower at the total hip or femoral neck and either one or more moderate or severe vertebral fractures or two or more mild vertebral fractures, or a BMD T-score of -2.0 or lower at the total hip or femoral neck and either two or more moderate or severe vertebral fractures or a fracture of the proximal femur sustained 3-24 months before randomization. Use of drugs affecting bone metabolism within defined washout periods was an exclusion criterion in the protocol. Patients enrolled in ARCH from Hong Kong, Republic of Korea, and Taiwan were included in this East Asian post hoc analysis.

The study was conducted in accordance with the Declaration of Helsinki, the Harmonised Tripartite Guideline for Good Clinical Practice from the International Conference on Harmonisation, and local laws and was approved by an independent ethics committee, research ethics board, or institutional review board for each study site (ClinicalTrials.gov NCT01631214). All patients provided written informed consent. Amgen provided financial support for the study.

\section{Assessment and procedures}

Assessments and procedures for patients enrolled in the study have been previously described in detail [27]. Briefly, lateral radiographs of the thoracic and lumbar spine were obtained at screening and months 12 and 24. Radiographs were assessed at a central imaging center, as were non-vertebral fractures. $\mathrm{BMD}$ at the lumbar spine and proximal femur were evaluated by dual-energy X-ray absorptiometry (DXA) at baseline and every 12 months thereafter. 
Adverse events (AEs) were reported by individual trial sites and summarized according to the Medical Dictionary for Regulatory Activities (MedDRA) version 19.1 coding system and the National Cancer Institute's Common Terminology Criteria for Adverse Events version 3.0 grading system. Serious cardiovascular AEs were adjudicated by the Duke Clinical Research Institute, and potential cases of osteonecrosis of the jaw and atypical femoral fracture were adjudicated by independent committees. Serum was tested for anti-romosozumab antibodies at day 1 and until month 24; samples that were positive for binding antibodies were assessed for neutralizing antibodies.

\section{Study endpoint}

The primary endpoints of the main study were the incidence of new vertebral fractures at 24 months and incidence of clinical fractures (non-vertebral and symptomatic vertebral fracture) at the time of the primary analysis (i.e., when clinical fracture events had been confirmed in at least 330 patients and all patients had the opportunity to complete the month 24 visit). Secondary endpoints included BMD at the lumbar spine, total hip, and femoral neck at 12 and 24 months, incidences of nonvertebral and hip fracture at the time of the primary analysis, and safety outputs, including adjudicated serious cardiovascular AEs, osteonecrosis of the jaw events, and atypical femoral fracture events.

\section{Statistical analysis}

This East Asia post hoc subanalysis of patients enrolled in Hong Kong, Republic of Korea, and Taiwan evaluated the same endpoints as the main ARCH study. The East Asia cohort analysis was not powered to determine statistical significance between groups for antifracture efficacy.

Analyses of vertebral fracture endpoints included all randomly assigned patients with a baseline radiograph and $\geq 1$ radiograph obtained post-baseline. Any missing post-baseline radiograph assessment was imputed using the status from the last non-missing post-baseline visit. Analyses of other fracture endpoints included all randomized patients. For the incidence of new vertebral and new or worsening vertebral fractures, risk ratios were determined based on the Mantel-Haenszel method, with treatment comparison assessed using a logistic-regression model stratified by age ( $<75 \mathrm{vs} \geq 75$ years), presence or absence of severe vertebral fracture at baseline, and baseline BMD T-score at the total hip $(\leq-2.5 \mathrm{vs}>-2.5)$. For other fracture endpoints, treatment groups were compared based on a Cox proportional-hazards model adjusted for age ( $<75$ vs $\geq 75$ years), presence or absence of severe vertebral fracture at baseline, and baseline BMD T-score at the total hip.

Percentage changes from baseline in BMD were assessed in patients who had a baseline and at least one post-baseline measurement. Between-group comparisons of BMD changes were analyzed using an analysis-of-covariance model with adjustment for age, severe vertebral fracture at baseline, baseline BMD, machine type, and baseline BMD-by-machinetype interaction, with missing values imputed by carrying forward the last post-baseline observation.

The safety analysis included all randomly assigned patients who received at least one dose of romosozumab or alendronate in the double-blind period. Incidence rates of AEs at the time of the primary analysis were cumulative and included events in the double-blind and in the open-label periods in patients who received at least one dose of open-label alendronate.

\section{Results}

\section{Patients}

Of 4093 patients randomized in the ARCH study, 275 were from Hong Kong, Republic of Korea, and Taiwan and were included in this East Asia subanalysis. The demographic and clinical characteristics of East Asian patients at baseline were balanced between the two treatment groups (Table 1) and were largely similar to those of the global ARCH population in terms of age and history of prior fracture, although the median Fracture Risk Assessment Tool (FRAX ${ }^{\circledR}$ ) score was higher in this subgroup (25.7) compared with the global population (17.9). The mean age of East Asian patients was 74.6 years, and more than half $(52.7 \%)$ were $\geq 75$ years old at enrollment. The mean body mass index (BMI) of East Asian patients was slightly lower than for the global population $(23.0 \mathrm{vs} 25.0 \mathrm{~kg}$ / $\mathrm{m}^{2}$ ). All patients had a previous osteoporotic fracture at age $\geq$ 45 years, and $98 \%$ had a prevalent vertebral fracture. Mean BMD T-scores were -3.0 at the lumbar spine, -2.8 at the total hip, and -3.1 at the femoral neck. Among East Asian patients randomized in the $\mathrm{ARCH}$ study, $10.5 \%$ had previously received osteoporosis medications outside of the respective defined washout periods.

\section{Efficacy}

\section{Fracture}

Among East Asian patients, treatment with romosozumab followed by alendronate reduced the risk of new vertebral fractures at 24 months by $60 \%$ compared with alendronate alone $(2.5 \%$ vs $6.6 \% ; P=0.11$; Fig. 1$)$. Data from the primary analysis showed that treatment with romosozumab followed by alendronate resulted in a $44 \%$ lower risk of clinical fracture than with alendronate alone $(7.0 \%$ vs $11.6 \% ; P=0.15$; Fig. 1$)$.

Reductions in risk of new vertebral fractures at 24 months among East Asian patients were generally similar to the last- 
Table 1 Demographics and baseline characteristics of East Asian patients in the ARCH study

\begin{tabular}{lcc}
\hline Characteristic & $\begin{array}{c}\text { Alendronate } \\
(n=146)\end{array}$ & $\begin{array}{l}\text { Romosozumab } \\
(n=129)\end{array}$ \\
\hline Mean \pm SD age, years & $74.0 \pm 6.7$ & $75.2 \pm 7.0$ \\
Age $\geq 75$ years, $n(\%)$ & $71.0(48.6)$ & $74.0(57.4)$ \\
Mean \pm SD BMI, kg/m ${ }^{2}$ & $23.5 \pm 3.1$ & $23.2 \pm 3.1$ \\
Mean \pm SD BMD T-score & & $-3.1 \pm 1.2$ \\
Lumbar spine & $-3.0 \pm 1.1$ & $-2.9 \pm 0.7$ \\
Total hip & $-2.8 \pm 0.7$ & $-3.1 \pm 0.5$ \\
Femoral neck & $-3.1 \pm 0.5$ & $129(100)$ \\
Previous osteoporosis fracture at $\geq 45$ years of age, $n(\%)$ & $146(100)$ & $127(98.4)$ \\
Prevalent vertebral fracture, $n(\%)$ & $143(97.9)$ & $3(2.3)$ \\
Grade of most severe vertebral fracture ${ }^{\mathrm{a}}$ & & $43(33.3)$ \\
Mild & $6(4.1)$ & $81(62.8)$ \\
Moderate & $36(24.7)$ & $40(31.0)$ \\
Severe & $101(69.2)$ & $11(8.5)$ \\
Previous non-vertebral fracture at $\geq 45$ years of age, $n(\%)^{\mathrm{b}}$ & $42(28.8)$ & $25.7(22.2-31.4)$ \\
Previous hip fracture, $n(\%)^{\mathrm{b}}$ & $15(10.3)$ & $17(13.2)$ \\
Median FRAX score $(\text { range })^{\mathrm{c}}$ & $25.6(22.0-32.4)$ & $15(11.6)$ \\
Prior osteoporosis medication use, $n(\%)$ & $12(8.2)$ & $1(0.8)$ \\
Oral bisphosphonate & $8(5.5)$ & $1(0.8)$ \\
Intravenous bisphosphonate & $0(0.0)$ & $2(1.6)$ \\
SERM & $1(0.7)$ & $(2.7)$ \\
PTH or PTH derivatives & $2(1.4)$ & \\
Other & & \\
\hline & & \\
\hline
\end{tabular}

${ }^{a}$ Assessed with the use of the Genant grading scale; ${ }^{b}$ excludes pathologic or high-trauma fracture; ${ }^{c}$ the countryspecific FRAX [28] score, developed by the World Health Organization (www.shef.ac.uk/frax/), indicates the 10year probability of major osteoporotic fracture, expressed as a percentage and calculated with BMD. PTH, parathyroid hormone; $S D$, standard deviation; SERM, selective estrogen receptor modulator

\section{a Incidence of new vertebral fractures through month 24}

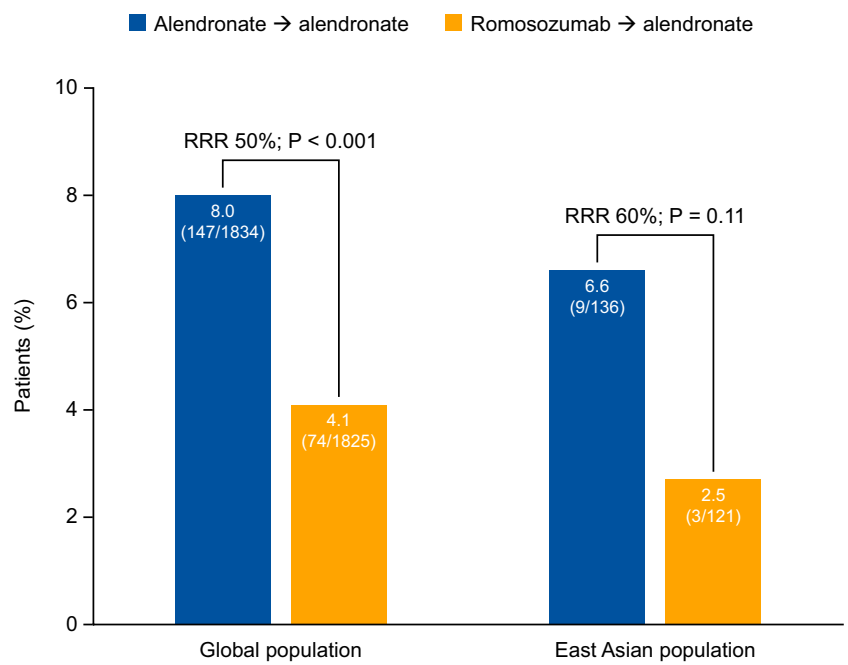

Fig. 1 Incidence of a new vertebral fractures through 24 months (LOCF imputation) and $\mathbf{b}$ clinical fracture at the time of the primary analysis* in the $\mathrm{ARCH}$ East Asian and Global populations. $\mathrm{n} / \mathrm{N}$ presented in bars $=$ number of patients with $\geq 1$ fracture/all randomly assigned patients (for

\section{b Incidence of clinical fractures at primary analysis*}

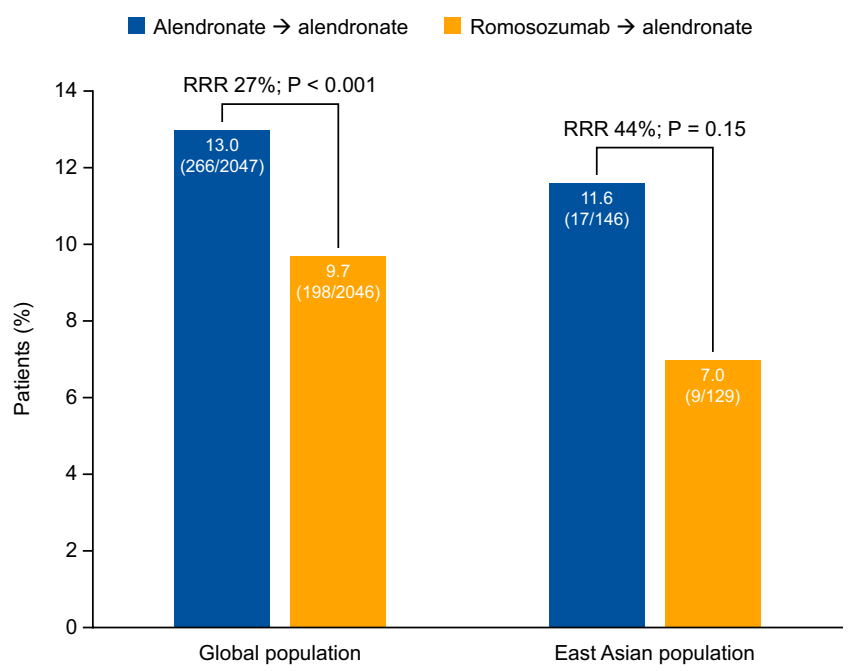

clinical fracture) or all randomly assigned patients with a baseline radiograph and $\geq 1$ radiograph obtained post-baseline (for new vertebral fractures). *Median follow-up at the time of primary analysis was 33 months (interquartile range 27-40). RRR relative risk reduction 
a Change in BMD at the lumbar spine

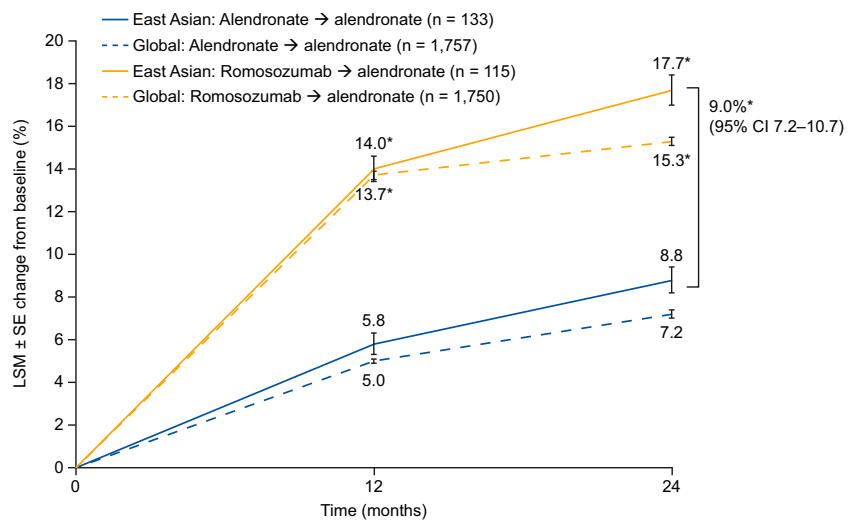

b Change in BMD at the total hip

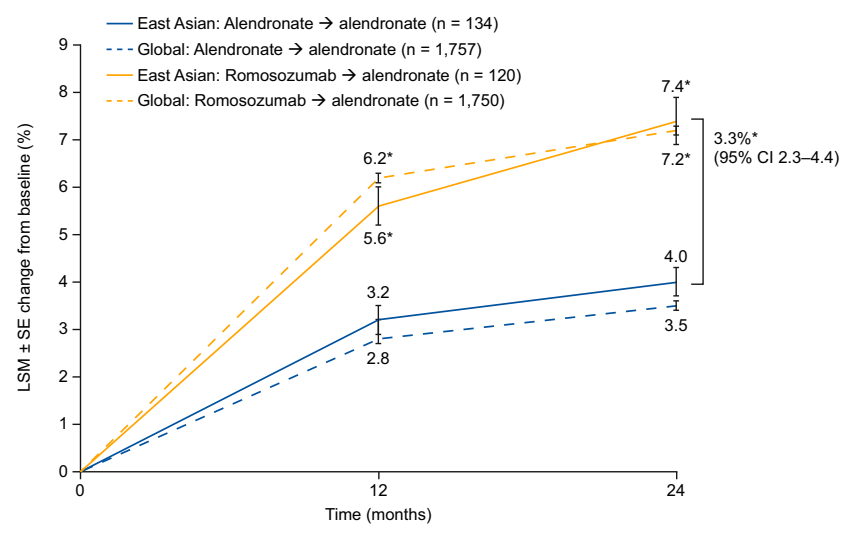

c Change in BMD at the femoral neck

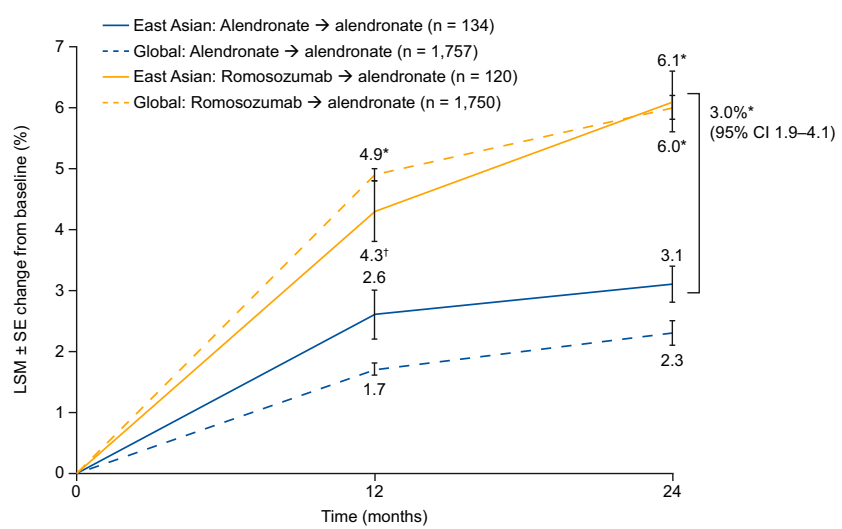

Fig. 2 Changes in BMD from baseline at months 12 and 24 among the ARCH East Asian and global populations in the a lumbar spine, b total hip, and c

femoral neck. $* P<0.001$ vs alendronate; ${ }^{\dagger} P=0.002 \mathrm{vs}$ alendronate. LSM least-squares mean, SE standard error

observation-carried-forward (LOCF) analysis of the global population (60\% vs 50\%; Fig. 1), although this finding did not reach statistical significance among East Asian patients as this analysis was not powered to demonstrate differences between subgroups.
At the time of the primary analysis, romosozumab followed by alendronate also resulted in a $60 \%$ lower risk of non-vertebral fracture than alendronate alone (95\% confidence interval [CI] $0.15-1.03 ; P=0.05)$, with fractures occurring in $4.7 \%(6 / 129)$ of patients in the romosozumab-to-alendronate group vs $10.3 \%$ $(15 / 146)$ of those in the alendronate-only group. Four patients $(2.7 \%)$ in the alendronate-only group had suffered a hip fracture by the time of the primary analysis, while none of the patients treated with romosozumab followed by alendronate did.

\section{Bone mineral density}

In both the global and East Asian populations, patients who received romosozumab had significantly greater percentage gains in BMD from baseline at all measured sites at both 12 and 24 months than patients who received alendronate alone (Fig. 2).

Among East Asian patients, romosozumab treatment achieved greater gains in mean BMD at the lumbar spine (8.2\%; 95\% CI 6.7-9.7, $P<0.001)$, total hip $(2.4 \%$; 95\% CI 1.4-3.3, $P<0.001)$, and femoral neck $(1.7 \%$; $95 \%$ CI $0.6-2.8$, $P=0.002)$ at month 12 compared with alendronate. After transitioning to alendronate, the significantly greater BMD increases gained with romosozumab compared with alendronate in the first year were maintained at 24 months at key skeletal sites. Initiating osteoporosis treatment with a year of romosozumab before alendronate achieved significantly greater percentage gains in mean BMD at 24 months from baseline by a further $9.0 \%(95 \%$ CI $7.2-10.7, P<0.001)$ at the lumbar spine, $3.3 \%$ (95\% CI 2.3-4.4, $P<0.001$ ) at the total hip, and $3.0 \%(95 \% \mathrm{CI} 1.9-4.1, P<0.001)$ at the femoral neck than with alendronate alone.

\section{Safety}

The majority of East Asian patients received all 12 doses of romosozumab, with a mean cumulative romosozumab exposure of $2289 \mathrm{mg}$ (range 210-2520 mg). Nine patients in each treatment group had an AE leading to study treatment discontinuation during the primary analysis period. Common AEs were largely similar between both treatment groups in the East Asian population, except back pain, musculoskeletal pain, pain in extremity, and falls, which occurred in more patients in the alendronate group, and upper respiratory tract infection and cough, which occurred in more patients in the romosozumab group (Table 2). In the East Asian population, hypersensitivity was reported in 19 (13.0\%) patients receiving alendronate and $22(17.1 \%)$ patients receiving romosozumab during the double-blind period, while in the global population it was reported in $118(5.9 \%)$ and $122(6.0 \%)$ patients in the alendronate and romosozumab groups, respectively. Among East Asian patients, injection-site reactions were reported in $17(11.6 \%)$ of those receiving alendronate and $21(16.3 \%)$ of 
those receiving romosozumab, while in the global population it was reported in $53(2.6 \%)$ and $90(4.4 \%)$ patients in the alendronate and romosozumab groups, respectively. It is important to note that the East Asian population in this study is substantially smaller than the overall global population. In the East Asian population, between-group differences for hypersensitivity events and injection-site reactions are 3 and 4 patients, respectively. The types of events for both hypersensitivity and injection-site reactions that were reported by the East Asian population were similar to that of the global population.

Among East Asian patients, two adjudicated serious cardiovascular AEs were observed in each treatment group during the double-blind period (cardiac ischemic events, $n=1$ in each group; cerebrovascular events, $n=1$ romosozumab group; heart failure, $n=1$ alendronate group; Table 2).
No adjudicated events of osteonecrosis of the jaw or atypical femoral fracture were reported in the romosozumab treatment arm throughout the double-blind period, while one of each event was positively adjudicated during the open-label period in the alendronate-only group.

Throughout the trial, binding anti-romosozumab antibodies were observed in $12.4 \%$ (16/129) of East Asian patients who received romosozumab, and the development of neutralizing antibodies occurred infrequently $(0.8 \%$ [1/129]). Consistent with findings from the global ARCH analysis, there was no evidence that anti-romosozumab antibodies had an impact on the efficacy or safety of romosozumab among East Asian patients.

Rates of AEs were generally similar across the romosozumab and alendronate groups (Table 2), similar to

Table 2 Adverse events in East Asian patients in the ARCH study

\begin{tabular}{|c|c|c|c|c|}
\hline \multirow[t]{2}{*}{ Event, $n(\%)$} & \multicolumn{2}{|c|}{ Month 12: double-blind period } & \multicolumn{2}{|c|}{ Primary analysis: double-blind and open-label period ${ }^{\mathrm{a}}$} \\
\hline & $\begin{array}{l}\text { Alendronate } \\
(n=146)\end{array}$ & $\begin{array}{l}\text { Romosozumab } \\
(n=129)\end{array}$ & $\begin{array}{l}\text { Alendronate to alendronate } \\
(n=146)\end{array}$ & $\begin{array}{l}\text { Romosozumab to alendronate } \\
(n=129)\end{array}$ \\
\hline Adverse event during treatment ${ }^{\mathrm{b}}$ & $126(86.3)$ & $115(89.1)$ & $135(92.5)$ & $121(93.8)$ \\
\hline Upper respiratory tract infection & $64(43.8)$ & $65(50.4)$ & $96(65.8)$ & $84(65.1)$ \\
\hline Back pain & $31(21.2)$ & $14(10.9)$ & $52(35.6)$ & $41(31.8)$ \\
\hline Musculoskeletal pain & $21(14.4)$ & $4(3.1)$ & $41(28.1)$ & $17(13.2)$ \\
\hline Arthralgia & $19(13.0)$ & $21(16.3)$ & $47(32.2)$ & $43(33.3)$ \\
\hline Fall & $19(13.0)$ & $11(8.5)$ & $51(34.9)$ & $36(27.9)$ \\
\hline Pain in extremity & $15(10.3)$ & $7(5.4)$ & $36(24.7)$ & $28(21.7)$ \\
\hline Cough & $9(6.2)$ & $14(10.9)$ & $25(17.1)$ & $31(24.0)$ \\
\hline Serious adverse event & $18(12.3)$ & $14(10.9)$ & $51(34.9)$ & $41(31.8)$ \\
\hline Adjudicated serious cardiovascular event ${ }^{\mathrm{c}}$ & $2(1.4)$ & $2(1.6)$ & $6(4.1)$ & $5(3.9)$ \\
\hline Cardiac ischemic event & $1(0.7)$ & $1(0.8)$ & $2(1.4)$ & $3(2.3)$ \\
\hline Cerebrovascular event & 0 & $1(0.8)$ & $2(1.4)$ & $1(0.8)$ \\
\hline Heart failure & $1(0.7)$ & 0 & $2(1.4)$ & 0 \\
\hline Death & 0 & 0 & 0 & $1(0.8)$ \\
\hline $\begin{array}{l}\text { Event leading to discontinuation of trial } \\
\text { regimen }\end{array}$ & $2(1.4)$ & $4(3.1)$ & $9(6.2)$ & $9(7.0)$ \\
\hline $\begin{array}{l}\text { Event leading to discontinuation of trial } \\
\text { participation }\end{array}$ & 0 & $1(0.8)$ & $3(2.1)$ & $5(3.9)$ \\
\hline \multicolumn{5}{|l|}{ Event of interest ${ }^{\mathrm{d}}$} \\
\hline Hypersensitivity & $19(13.0)$ & $22(17.1)$ & $29(19.9)$ & $29(22.5)$ \\
\hline Injection-site reaction & $17(11.6)$ & $21(16.3)$ & $17(11.6)$ & $21(16.3)$ \\
\hline Osteoarthritis $^{\mathrm{e}}$ & $12(8.2)$ & $6(4.7)$ & $19(13.0)$ & $19(14.7)$ \\
\hline Cancer & 0 & $4(3.1)$ & $6(4.1)$ & $7(5.4)$ \\
\hline Hyperostosis $^{\mathrm{f}}$ & $1(0.7)$ & 0 & $2(1.4)$ & 0 \\
\hline Hypocalcemia & 0 & $1(0.8)$ & 0 & $1(0.8)$ \\
\hline Osteonecrosis of the jaw ${ }^{\mathrm{c}}$ & 0 & 0 & $1(0.7)$ & 0 \\
\hline Atypical femoral fracture ${ }^{c}$ & 0 & 0 & $1(0.7)$ & 0 \\
\hline
\end{tabular}

${ }^{a}$ Incidence rates were cumulative and included all events in the double-blind and open-label period (to 27 February 2017) in patients who received $\geq 1$ dose of open-label alendronate; ${ }^{b}$ events that occurred in $\geq 10 \%$ of patients in either group during the double-blind period; ${ }^{\mathrm{c}}$ serious cardiovascular adverse events were adjudicated by the Duke Clinical Research Institute, and potential cases of osteonecrosis of the jaw and atypical femoral fracture were adjudicated by independent committees; ${ }^{\mathrm{d}}$ identified by prespecified MedDRA search strategies; ${ }^{\mathrm{e}}$ prespecified events reported under the osteoarthritis search strategy were osteoarthritis, spinal osteoarthritis, and arthritis; ${ }^{\mathrm{f}}$ prespecified events reported under the hyperostosis search strategy were spinal column stenosis and vertebral foraminal stenosis 
rates seen in the global population [27], and consistent with the known safety profile of romosozumab.

\section{Discussion}

In this post hoc analysis of ARCH, East Asian postmenopausal women with severe osteoporosis and at high fracture risk who were treated with romosozumab for 12 months before receiving alendronate experienced greater reductions in fracture risk and significantly increased BMD at all sites compared with those who received just alendronate, a current standard of care for osteoporosis treatment. These findings are consistent with those observed in the overall ARCH population, which confirmed that the efficacy of romosozumab is superior to that of alendronate in postmenopausal women with osteoporosis at high risk of fracture [27].

Even though this subanalysis was not powered to determine treatment effects between subgroups, its findings are important, timely, and clinically relevant for Asia. Alendronate, the most widely used anti-osteoporosis agent in Asia [14-16], is an efficacious drug that was outperformed by romosozumab in a well-designed, global, phase III study [27]. $\mathrm{ARCH}$ is one of the first registrational studies of a bonebuilding agent vs an antiresorptive therapy designed with fracture prevention as the primary endpoint [27]. Compared with the placebo-controlled FRAME study, ARCH enrolled patients with more severe osteoporosis or higher fracture risk at baseline, a population with a current unmet medical need that requires rapid and potent antifracture efficacy. The East Asian subpopulation enrolled in ARCH closely reflects patients seen in real-life clinical practice in Asia.

As with the global ARCH population, reduced fracture risk among East Asian patients was observed in the first year of therapy, highlighting the ability of romosozumab to offer potent and rapid fracture prevention, as well as BMD gain benefits, which are key aspects important for practice. These findings may be especially important in Asia, where osteoporosis remains largely underdiagnosed and undertreated, and where the burden of imminent risk of fracture continues to increase $[8,9,29]$. Patients in Asia may have their osteoporosis identified later in the disease course, creating a need for a therapy that offers potent and rapid antifracture benefit to high-risk patients. In addition, clinical decisions are not regularly informed by bone-density assessments as access to DXA scans is limited in the region. As such, antifracture efficacy of osteoporosis treatments, as informed by fracture risk reduction, is especially helpful in the decision-making process for clinicians in Asia.

Romosozumab therapy was well tolerated in East Asian patients, and the safety profile was generally consistent with that for the global population of patients with postmenopausal osteoporosis. Rates of injection-site reaction and hypersensitivity, which are known adverse reactions with romosozumab, were higher in East Asian patients in both treatment arms compared with the global population and appear to have been driven by the smaller sample size in the East Asian population; however, rates of treatment discontinuation were similar to those of the overall population, suggesting that AEs were manageable. While an imbalance in positively adjudicated serious cardiovascular AEs was observed in the overall ARCH population (50 vs 38 cases at 12 months), the incidence rates in the East Asian subgroup were balanced, occurring in only two patients per treatment arm at 12 months, although the sample size may be too small to draw meaningful conclusions.

A limitation of the current post hoc analysis is that $\mathrm{ARCH}$ was not powered to detect differences in treatment effect in the East Asia subgroup. The heterogeneity of ethnicities across East Asia also precludes generalizing these results for the rest of Asia.

Nevertheless, findings from this subanalysis add to previous published findings of romosozumab clinical studies in the Asia Pacific region. Prior studies have shown that romosozumab provides similar pharmacokinetic attributes between healthy global and Japanese postmenopausal populations (study 20090378), as well as similar dose-response profiles between global and East Asian postmenopausal populations in phase II (20060326 and Ishibashi et al., 2017 [30]) and phase III studies (20070337). In postmenopausal Japanese women with osteoporosis, romosozumab treatment has been shown to lead to large and significant BMD gains [30] and substantial reductions in fracture risk [31] compared with placebo. Taken together, these studies suggest no clinically significant interethnic difference in the effects of romosozumab between the global population and patients in East Asia for the treatment of osteoporosis in postmenopausal women at high fracture risk.

\section{Conclusions}

Among East Asian patients, rapid BMD gains from boneforming therapy with romosozumab followed by alendronate were associated with a lower risk of new vertebral, clinical, non-vertebral, and hip fractures than with alendronate alone over the course of 2 years of treatment. Romosozumab treatment was well tolerated in East Asian patients; AEs were generally similar to those observed in the global population and consistent with the known safety profile of romosozumab. The imbalance in positively adjudicated serious cardiovascular AEs seen in the overall ARCH population was not observed in the East Asian subgroup, although the sample size may be too small to draw meaningful conclusions. The impressive fracture risk reductions observed with romosozumab treatment in both the global and East Asian populations 
confirm the benefits of initiating treatment with an anabolic agent followed by an antiresorptive compared with starting with antiremodeling therapy, especially in patients at high or imminent risk of fracture.

Acknowledgments The authors would like to thank all patients who participated in the study and clinical personnel involved in data collection. Writing assistance was provided by Stefanie Chuah and Helene Wellington from AMICULUM, funded by Amgen, Inc.

Funding information This study was funded by Amgen, Inc., Astellas Pharma, and UCB Pharma.

\section{Compliance with ethical standards}

Conflicts of interest CW has received honoraria from Amgen. JM, CT, and WY are employees of Amgen. RD was an employee of Amgen at the time of manuscript development. JG is an employee of UCB Pharma. No other potential conflict of interest relevant to the article was reported.

Data sharing statement Qualified researchers may request data from Amgen clinical studies. Complete details are available at http://www. amgen.com/datasharing.

Ethical approval All procedures performed in studies involving human participants were in accordance with the ethical standards of the institutional and/or national research committee and with the 1964 Helsinki declaration and its later amendments or comparable ethical standards.

Informed consent Informed consent was obtained from all individual participants included in the study.

Open Access This article is licensed under a Creative Commons Attribution-NonCommercial 4.0 International License, which permits any non-commercial use, sharing, adaptation, distribution and reproduction in any medium or format, as long as you give appropriate credit to the original author(s) and the source, provide a link to the Creative Commons licence, and indicate if changes were made. The images or other third party material in this article are included in the article's Creative Commons licence, unless indicated otherwise in a credit line to the material. If material is not included in the article's Creative Commons licence and your intended use is not permitted by statutory regulation or exceeds the permitted use, you will need to obtain permission directly from the copyright holder. To view a copy of this licence, visit http:// creativecommons.org/licenses/by-nc/4.0/.

\section{References}

1. Cummings SR, Melton LJ (2002) Epidemiology and outcomes of osteoporotic fractures. Lancet 359:1761-1767. https://doi.org/10. 1016/S0140-6736(02)08657-9

2. Fukushima T, Sudo A, Uchida A (2006) Bilateral hip fractures. J Orthop Sci 11:435-438. https://doi.org/10.1007/s00776-006-10563

3. International Osteoporosis Foundation (2013) The Asia-Pacific regional audit: epidemiology, costs and burden of osteoporosis in 2013. Nyon, Switzerland

4. Cho HM, Lee K, Min W, Choi YS, Lee HS, Mun HJ, Shim HY, Lee DG, Yoo MJ (2016) Survival and functional outcomes after hip fracture among nursing home residents. J Korean Med Sci 31:8997. https://doi.org/10.3346/jkms.2016.31.1.89

5. Johansson H, Siggeirsdóttir K, Harvey NC, Odén A, Gudnason V, McCloskey E, Sigurdsson G, Kanis JA (2017) Imminent risk of fracture after fracture. Osteoporos Int 28:775-780. https://doi.org/ 10.1007/s00198-016-3868-0

6. Bonafede M, Shi N, Barron R, Li X, Crittenden DB, Chandler D (2016) Predicting imminent risk for fracture in patients aged 50 or older with osteoporosis using US claims data. Arch Osteoporos 11: 26. https://doi.org/10.1007/s11657-016-0280-5

7. Roux C, Briot K (2017) Imminent fracture risk. Osteoporos Int 28: 1765-1769. https://doi.org/10.1007/s00198-017-3976-5

8. Ghazi MA, Sufyan M, Alliuddin A et al (2017) Prevention of subsequent fractures of hip with use of bisphosphonates in Pakistan population. Osteoporos Sarcopenia 3:S36. https://doi.org/10.1016/ j.afos.2017.08.066

9. Hagino H, Sawaguchi T, Endo N, Ito Y, Nakano T, Watanabe Y (2012) The risk of a second hip fracture in patients after their first hip fracture. Calcif Tissue Int 90:14-21. https://doi.org/10.1007/ s00223-011-9545-6

10. Chen F-P, Shyu Y-C, Fu T-S, Sun CC, Chao AS, Tsai TL, Huang TS (2017) Secular trends in incidence and recurrence rates of hip fracture: a nationwide population-based study. Osteoporos Int 28:811818. https://doi.org/10.1007/s00198-016-3820-3

11. Gullberg B, Johnell O, Kanis JA (1997) World-wide projections for hip fracture. Osteoporos Int 7:407-413. https://doi.org/10.1007/ PL00004148

12. Cheung C-L, Ang SB, Chadha M et al (2018) An updated hip fracture projection in Asia: the Asian Federation of Osteoporosis Societies study. Osteoporos Sarcopenia 4:16-21. https://doi.org/10. 1016/j.afos.2018.03.003

13. Kung AW, Fan T, Xu L, Xia WB, Park IH, Kim HS, Chan SP, Lee JK, Koh L, Soong YK, Soontrapa S, Songpatanasilp T, Turajane T, Yates M, Sen S (2013) Factors influencing diagnosis and treatment of osteoporosis after a fragility fracture among postmenopausal women in Asian countries: a retrospective study. BMC Womens Health 13:7. https://doi.org/10.1186/1472-6874-13-7

14. Man Y, Pan W, Lu J, Li SY, Zhang P, Guo JZ, Yu SF, Zhang B, Xiao E, Zhang C, Zuo W, Jin HJ, Chen Y, Wu B, Shi XE, Hong D, Liu X, Gao YX, Li J, Yin L, Xue QY (2016) Treatment and management of osteoporotic fractures: a nation-wide survey of 484 senior orthopaedists in China. Orthop Surg 8:432-439. https://doi.org/ 10.1111/os.12296

15. Suzuki N, Arai K, Kon S, Yamanaka K, Otsuka H, Koizumi M, Hosaka N, Tsuchiya M, Mochizuki T, Kuraishi T, Murayama T, Tashi H, Oike N, Wakasugi M, Takahashi Y, Nakadai M, Endo N (2017) Challenges to prevent secondary fractures in patients with hip fractures in Joetsu Myoko, Japan through the increased use of osteoporosis treatment and collaboration with family doctors. J Bone Miner Metab 35:315-323. https://doi.org/10.1007/s00774016-0758-7

16. Yeap SS, Nur Fazirah MFR, Nur Aisyah C, Zahari Sham SY, Samsudin IN, C Thambiah S, Hew FL, Lim BP, Siow YS, Chan SP (2017) Trends in post osteoporotic hip fracture care from 2010 to 2014 in a private hospital in Malaysia. Osteoporos Sarcopenia 3: 112-116. https://doi.org/10.1016/j.afos.2017.05.001

17. Kim SC, Kim M, Sanfélix-Gimeno G et al (2015) Use of osteoporosis medications after hospitalization for hip fracture: a crossnational study. Am J Med 128:519-526.e1. https://doi.org/10. 1016/j.amjmed.2015.01.014

18. Wu X, Wei D, Sun B, Wu XN (2016) Poor medication adherence to bisphosphonates and high self-perception of aging in elderly female patients with osteoporosis. Osteoporos Int 27:3083-3090. https:// doi.org/10.1007/s00198-016-3763-8

19. Hsu C-Y, Chiu W-C, Chen J-F, Chou CL, Su YJ, Yu SF, Cheng TT (2015) Medical specialty-related adherence to anti-osteoporotic 
regimens in fragility hip fracture patients. J Bone Miner Metab 33: 577-583. https://doi.org/10.1007/s00774-014-0621-7

20. Cheung EYN, Tan KCB, Cheung C-L, Kung AWC (2016) Osteoporosis in East Asia: current issues in assessment and management. Osteoporos Sarcopenia 2:118-133. https://doi.org/10. 1016/j.afos.2016.07.001

21. Mithal A, Kaur P (2012) Osteoporosis in Asia: a call to action. Curr Osteoporos Rep 10:245-247. https://doi.org/10.1007/s11914-0120114-3

22. Padhi D, Jang G, Stouch B, Fang L, Posvar E (2011) Single-dose, placebo-controlled, randomized study of AMG 785, a sclerostin monoclonal antibody. J Bone Miner Res 26:19-26. https://doi.org/ $10.1002 / j b m r .173$

23. McClung MR, Grauer A, Boonen S, Bolognese MA, Brown JP, Diez-Perez A, Langdahl BL, Reginster JY, Zanchetta JR, Wasserman SM, Katz L, Maddox J, Yang YC, Libanati C, Bone HG (2014) Romosozumab in postmenopausal women with low bone mineral density. N Engl J Med 370:412-420. https://doi.org/ 10.1056/NEJMoa1305224

24. McClung MR (2018) Romosozumab for the treatment of osteoporosis. Osteoporos Sarcopenia 4:11-15. https://doi.org/10.1016/j. afos.2018.03.002

25. Cosman F, Crittenden DB, Adachi JD et al (2016) Romosozumab treatment in postmenopausal women with osteoporosis. N Engl J Med 375:1532-1543. https://doi.org/10.1056/NEJMoa1607948

26. Pharmaceuticals and Medical Devices Agency. Prescription drug database: romosozumab (genetically modified) [in Japanese]. http://www.pmda.go.jp/PmdaSearch/iyakuDetail/GeneralList/ 3999449G1

27. Saag KG, Petersen J, Brandi ML, Karaplis AC, Lorentzon M, Thomas T, Maddox J, Fan M, Meisner PD, Grauer A (2017)
Romosozumab or alendronate for fracture prevention in women with osteoporosis. N Engl J Med 377:1417-1427. https://doi.org/ 10.1056/NEJMoa1708322

28. Kanis JA, Hans D, Cooper C, Baim S, Bilezikian JP, Binkley N, Cauley JA, Compston JE, Dawson-Hughes B, el-Hajj Fuleihan G, Johansson H, Leslie WD, Lewiecki EM, Luckey M, Oden A, Papapoulos SE, Poiana C, Rizzoli R, Wahl DA, McCloskey E, Task Force of the FRAX Initiative (2011) Interpretation and use of FRAX in clinical practice. Osteoporos Int 22:2395-2411. https://doi.org/10.1007/s00198-011-1713-z

29. Lee SH, Chen IJ, Li YH, Fan Chiang CY, Chang CH, Hsieh PH (2016) Incidence of second hip fractures and associated mortality in Taiwan: a nationwide population-based study of 95,484 patients during 2006-2010. Acta Orthop Traumatol Turc 50:437-442. https://doi.org/10.1016/j.aott.2016.06.008

30. Ishibashi H, Crittenden DB, Miyauchi A, Libanati C, Maddox J, Fan M, Chen L, Grauer A (2017) Romosozumab increases bone mineral density in postmenopausal Japanese women with osteoporosis: a phase 2 study. Bone 103:209-215. https://doi.org/10.1016/j. bone.2017.07.005

31. Miyauchi A, Dinavahi R, Crittenden D, Yang W, Maddox JC, Hamaya E, Nakamura Y, Libanati C, Grauer A, Shimauchi J (2019) Increased bone mineral density for 1 year of romosozumab, vs placebo, followed by 2 years of denosumab in the Japanese subgroup of the pivotal FRAME trial and extension. Arch Osteoporos 14:59. https://doi.org/10.1007/s11657-019-0608-z

Publisher's note Springer Nature remains neutral with regard to jurisdictional claims in published maps and institutional affiliations. 